\title{
TREE COVER NUMBER AND MAXIMUM SEMIDEFINITE NULLITY OF SOME GRAPH CLASSES*
}

\author{
RACHEL DOMAGALSKI ${ }^{\dagger}$ AND SIVARAM K. NARAYAN ${ }^{\ddagger}$
}

\begin{abstract}
Let $G$ be a graph with a vertex set $V$ and an edge set $E$ consisting of unordered pairs of vertices. The tree cover number of $G$, denoted $\tau(G)$, is the minimum number of vertex disjoint simple trees occurring as induced subgraphs of $G$ that cover all the vertices of $G$. In this paper, the tree cover number of a line graph $\tau(L(G))$ is shown to be equal to the path number $\pi(G)$ of $G$. Also, the tree cover numbers of shadow graphs, corona and Cartesian product of two graphs are found.

The graph parameter $\tau(G)$ is related to another graph parameter $M_{+}(G)$, called the maximum semidefinite nullity of $G$. Suppose $S_{+}(G, \mathbb{R})$ denotes the collection of positive semidefinite real symmetric matrices associated with a given graph $G$. Then $M_{+}(G)$ is the maximum nullity among all matrices in $S_{+}(G, \mathbb{R})$. It has been conjectured that $\tau(G) \leq M_{+}(G)$. The conjecture is shown to be true for graph classes considered in this work.
\end{abstract}

Key words. Tree cover number, Line graphs, Shadow graphs, Corona graphs, Cartesian product graphs, Maximum semidefinite nullity.

AMS subject classifications. 05C50, 15A03, 15A18.

1. Introduction. Let $G=(V, E)$ denote a simple graph with vertex set $V=\left\{v_{1}, v_{2}, \ldots, v_{n}\right\}$ and edge set $E$ consisting of two-element subsets of $V$. The number of vertices of $G$ is called the order of $G$ and is denoted $|G|$. If there are $m$ edges in $G$, then the graph is said to have size $m$. A graph parameter we will be discussing in this paper is called the tree cover number of $G$, denoted $\tau(G)$. We define $\tau(G)$ to be the minimum number of vertex disjoint simple trees occurring as induced subgraphs of $G$ that cover all the vertices of $G$. This parameter was introduced in [2] and it was shown that the maximum semidefinite nullity of an outerplanar graph is equal to its tree cover number.

In this paper, we will find the tree cover number of some graph classes. For a simple connected graph $G$, we prove that the tree cover number of its line graph $L(G)$ is equal to the minimum number of paths $\pi(G)$ needed in a path decomposition of $G$. Thus, the tree cover number of a line graph $L(G)$ is related to the well studied parameter of path number of $G$. We then study the tree cover number of the shadow graph $S(G)$ of a simple graph $G$. For given simple graphs $G$ and $H$ we find the tree cover number of the corona $G \circ H$, and the Cartesian product $G \square H$.

Given an $n \times n$ Hermitian matrix $A$, its graph $G(A)$ is the simple graph on $n$ vertices $\left\{v_{1}, v_{2}, \ldots, v_{n}\right\}$, which has an edge between $v_{i}$ and $v_{j}$ if and only if the $(i, j) t h$ entry of $A$ is nonzero and $i \neq j$. The graph is independent of the real diagonal entries of $A$. The set of real symmetric positive semidefinite matrices that share a common graph $G$ is denoted $S_{+}(G, \mathbb{R})$. We define the minimum semidefinite rank of $G, m r_{+}^{\mathbb{R}}(G)$, as $\min \left\{\operatorname{rank} A: A \in S_{+}(G, \mathbb{R})\right\}$ and the maximum semidefinite nullity of $G, M_{+}^{\mathbb{R}}(G)$, as $\max \{$ nullity $A$ : $\left.A \in S_{+}(G, \mathbb{R})\right\}$. Similar parameters $m r_{+}^{\mathbb{C}}(G)$ and $M_{+}^{\mathbb{C}}(G)$ are defined by considering the complex Hermitian positive semidefinite matrices $S_{+}(G, \mathbb{C})$ associated with a given graph $G$. Since $S_{+}(G, \mathbb{R}) \subseteq S_{+}(G, \mathbb{C})$ it is

*Received by the editors on March 23, 2020. Accepted for publication on August 5, 2020. Handling Editor: Michael Tsatsomeros. Corresponding Author: Rachel Domagalski.

${ }^{\dagger}$ Department of Mathematics, Michigan State University, East Lansing, MI (domagal9@msu.edu).

¥Department of Mathematics, Central Michigan University, Mount Pleasant, MI (sivaram.narayan@cmich.edu). 
Electronic Journal of Linear Algebra, ISSN 1081-3810

A publication of the International Linear Algebra Society

Volume 36, pp. 678-693, September 2020.

clear that $m r_{+}^{\mathbb{C}}(G) \leq m r_{+}^{\mathbb{R}}(G)$ and $M_{+}^{\mathbb{R}}(G) \leq M_{+}^{\mathbb{C}}(G)$. It has been shown that strict inequality $m r_{+}^{\mathbb{C}}(G)<$ $m r_{+}^{\mathbb{R}}(G)$ is possible [1]. Since we mainly consider the graph parameter tree cover number of $G$, our results hold for both real and complex fields. We therefore omit the field reference and write $m r_{+}(G)$ and $M_{+}(G)$ instead.

In [2], it has been shown that $\tau(G) \leq M_{+}(G)$ for chordal graphs and certain bipartite graphs and that the equality holds for outerplanar graphs. It has been conjectured that $\tau(G) \leq M_{+}(G)$ for every graph $G$. Each section of this paper examines a different class of graph: line graphs, shadow graphs, corona graphs, and Cartesian products. At the end of each section, we discuss the connection between the newly found tree cover number and their corresponding maximum semidefinite nullity.

2. Preliminaries. A graph $G$ is simple if it contains no loops or parallel edges. We write $e=u v$ for an edge $e$ with endpoints $u$ and $v$. If $u$ and $v$ are the ends of an edge, they are said to be adjacent or neighbors. The ends of an edge are said to be incident with the edge, and vice-versa. The set of neighbors of a vertex $v$ in $G$ is denoted $N_{G}(v)$. The number of edges of $G$ incident with $v$ is called the degree of $v$ in $G$, denoted $d_{G}(v)$. For a simple graph, $d_{G}(v)=\left|N_{G}(v)\right|$. A vertex of degree one is called a pendant vertex or a leaf of $G$.

Given a graph $G, F$ is a subgraph of $G$ if $V(F) \subseteq V(G)$ and $E(F) \subseteq E(G)$. An induced subgraph of $G$, denoted by $G[Y]$, is the subgraph of $G$ whose vertex set is $Y$ and whose edge set consists of all edges in $E(G)$ which have both ends in $Y$.

A path is a simple graph whose vertices can be arranged in a linear sequence $v_{1} v_{2} \cdots v_{n}$ such that two vertices are adjacent if and only if they are consecutive in the sequence. The path on $n$ vertices is denoted $P_{n}$. A graph $G$ is connected if there exists a path between any pair of vertices. A cycle on three or more vertices is a simple graph whose vertices can be arranged in a cyclic sequence so that two vertices are adjacent if they are consecutive in the sequence and nonadjacent otherwise. The cycle on $n$ vertices is denoted by $C_{n}$. Note that $\tau\left(C_{n}\right)=2$, where one tree is a single vertex $v$ in $C_{n}$ and a second tree is the path induced by $V\left(C_{n}\right) \backslash v$

A graph with no cycle is called acyclic. A tree is a connected acyclic graph. One type of tree is called a star, denoted $S t_{n}$, which has one vertex of degree $n-1$ and the remaining vertices are pendant vertices. For any tree $G$, by definition $\tau(G)=1$.

A complete graph $K_{n}$ on $n$ vertices is a simple graph where any two vertices are adjacent. Since any three vertices of $K_{n}$ induce a cycle, each tree in a minimum tree cover of $K_{n}$ can only contain two vertices. Hence, $\tau\left(K_{n}\right)=\left\lceil\frac{n}{2}\right\rceil$. A graph is said to be bipartite if its vertex set can be partitioned into two subsets $X$ and $Y$, called parts, so that every edge has one end in $X$ and one end in $Y$. If every vertex in $X$ is joined to every vertex in $Y$, then $G$ is called a complete bipartite graph, denoted $K_{m, n}$ where $|X|=m$ and $|Y|=n$. For a complete bipartite graph, a minimum tree cover can be constructed by letting one induced tree contain a single vertex from partite set $X$, and all but one vertex from partite set $Y$. The remaining vertices induce the second tree. Hence, $\tau\left(K_{m, n}\right)=2$.

An independent set in a graph is a set of pairwise nonadjacent vertices. The maximum cardinality among independent sets in $G$ is called the independence number, denoted by $\alpha(G)$. A clique of a graph $G$ is a set of mutually adjacent vertices. The minimum number of cliques needed to cover all single edges of a graph $G$ is called the clique cover number of $G$, denoted $c c(G)$. 
The contraction of an edge $e=u v$ involves the deletion of $e$ and merging of the vertices $u$ and $v$ into a new vertex $w$, keeping all the edges in $G$ incident to either $u$ or $v$. A minor of a graph $G$ is any graph obtainable from $G$ by means of a sequence of vertex and edge deletions and edge contractions [5, p. 268].

A graph $G$ is said to be chordal if it has no induced cycles of length four or more. A graph which can be drawn in the plane in such a way that the edges meet only at points corresponding to their common ends is called a planar graph, and such a drawing is called a planar embedding of the graph. A graph is outerplanar if it has a crossing-free planar embedding such that all vertices are on the same face.

Given a graph $G$ and $v \in V(G)$, the orthogonal removal of $v$ from $G$, denoted $G \ominus v$, is a multigraph modified from $G[V(G)-\{v\}]$ by adding $P(u, w)$ additional edges between each pair $u, w \in N_{G}(v)$ where $P(u, w)$ is the product of the number of edges from $v$ to $u$ and from $v$ to $w$. For further details in graph theory, one may consult [5] or [19].

We are interested in considering graphs associated to real symmetric or complex Hermitian matrices. The set of $n \times n$ matrices with entries that are complex numbers is denoted by $M_{n}(\mathbb{C})$ and those with entries that are real numbers is denoted by $M_{n}(\mathbb{R})$. A matrix $A \in M_{n}(\mathbb{C})$ is said to be Hermitian if $A$ equals its conjugate transpose $A^{*}$. A matrix $A \in M_{n}(\mathbb{R})$ is said to be symmetric if $A$ equals its transpose $A^{T}$.

If $\vec{x}=\left(x_{1}, x_{2}, \ldots, x_{n}\right)$ and $\vec{y}=\left(y_{1}, y_{2}, \ldots, y_{n}\right)$ are points in $\mathbb{C}^{n}$, then the Euclidean inner product $\langle\vec{x}, \vec{y}\rangle=\sum_{i=1}^{n} x_{i} \overline{y_{i}}$. A Hermitian matrix $A \in M_{n}(\mathbb{C})$ is positive definite if $\langle A \vec{x}, \vec{x}\rangle$ is positive for all nonzero $\vec{x}$ in $\mathbb{C}^{n}$ and it is positive semidefinite if $\langle A \vec{x}, \vec{x}\rangle \geq 0$ for all $\vec{x}$ in $\mathbb{C}^{n}$.

We may think of $A \in M_{m, n}(\mathbb{C})$ as a linear transformation $\vec{x} \mapsto A \vec{x}$ from $\mathbb{C}^{n}$ to $\mathbb{C}^{m}$. We define for $A \in M_{m, n}(\mathbb{C})$, the nullspace of $A$ and the range of $A$ as follows: nullspace $A=\left\{\vec{x} \in \mathbb{C}^{n}: A \vec{x}=0\right\}$ and range $A=\left\{\vec{y} \in \mathbb{C}^{m}: \vec{y}=A \vec{x}\right.$ for some $\left.\vec{x} \in \mathbb{C}^{n}\right\}$. The dimension of the nullspace $A$ is called the nullity of $A$ and the dimension of the range $A$ is called the rank of $A$. These numbers are related by the rank-nullity theorem: $\operatorname{rank} A+$ nullity $A=n$.

Let $A$ be a real symmetric $n \times n$ matrix. Then $A$ is said to satisfy the Strong Arnold Property if there does not exist a $n \times n$ symmetric matrix $x \neq 0$ such that (i) $A x=0$, (ii) $A \circ x=0$, and (iii) $I \circ x=0$ where $\circ$ denotes the entrywise (Hadamard) product and $I$ is the identity matrix.

Define the parameter $\nu(G)$ to be the maximum nullity among matrices $A \in S_{+}(G, \mathbb{R})$ that satisfy the Strong Arnold Property [9, 10]. For further results in Matrix Theory, one may consult [13].

3. Tree cover number of line graphs. Let $G$ be a simple connected graph with $n$ vertices and $m$ edges. The line graph of a graph $G$, denoted $L(G)$, has vertex set consisting of the $m$ edges of $G$ and an edge is drawn between two vertices in $L(G)$ if and only if the corresponding edges in $G$ have a common end vertex.

We present some well-known conditions for deciding when a graph $H$ is the line graph of a graph $G$. In order to state the result we need the following definitions.

A claw in a graph $G$ is defined to be an induced subgraph isomorphic to $K_{1,3}$. A triangle graph $S$ in $G$ is a subgraph isomorphic to $K_{3}$. A triangle $S$ in $G$ is odd if $|N(v) \cap V(S)|$ is odd for some $v \in V(G)$. A triangle $S$ in $G$ is even if $|N(v) \cap V(S)|$ is even for every $v \in V(G)$ [19]. 
Electronic Journal of Linear Algebra, ISSN 1081-3810

A publication of the International Linear Algebra Society

Volume 36, pp. 678-693, September 2020 .

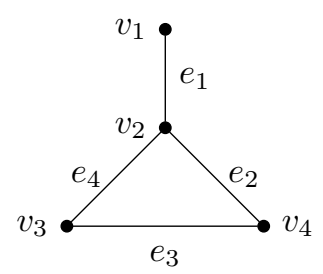

$G$

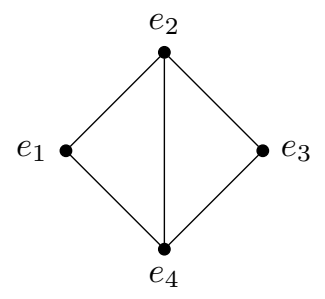

$H$

Figure 1. $H$ is the line graph of $G$.

Theorem 3.1. ([[3, 4, 14, 18]]) Let $H$ be a graph. The following conditions are equivalent:

1. $H$ is a line graph of $G$; that is, $L(G)=H$;

2. The edges of $H$ can be partitioned into complete subgraphs such that no vertex lies in more than two of the subgraphs;

3. $H$ is claw-free and if two odd triangles have a common edge, then the subgraph induced by their vertices is a $K_{4}$;

4. $H$ contains none of the nine forbidden graphs of Figure 2 as an induced subgraph.
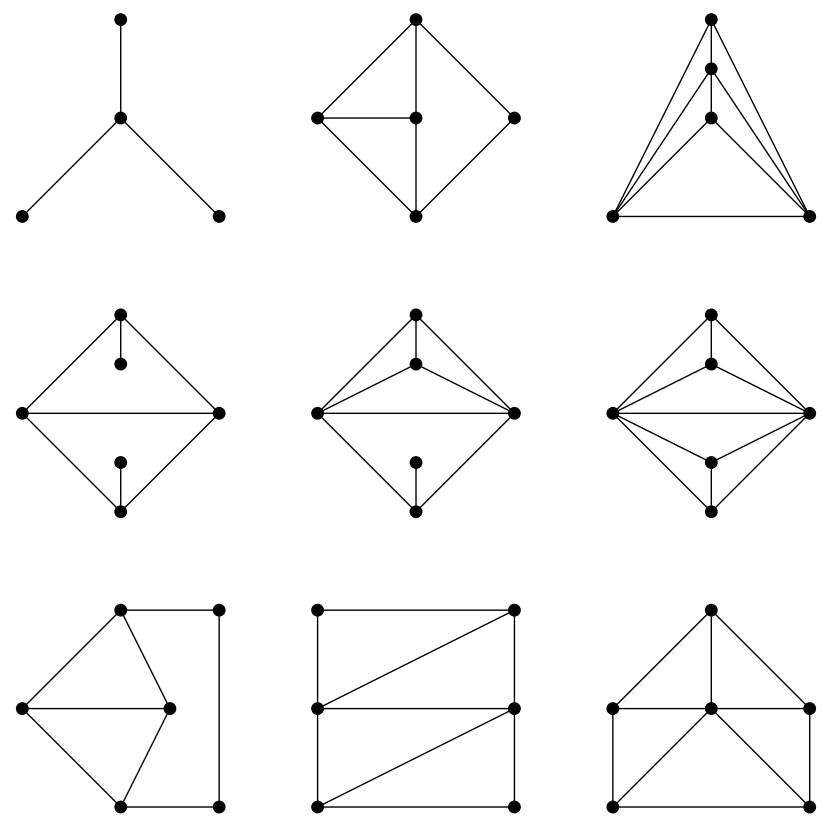

FigURE 2. These nine graphs are forbidden induced subgraphs of any line graph.

In order to find the tree cover number of $L(G)$, we first show that every induced simple tree in $L(G)$ is a path.

Proposition 3.2. Let $L(G)$ be the line graph of a simple graph $G$. Then the following hold:

1. Every induced simple tree in $L(G)$ must be an induced path obtained from a path in $G$ 
2. Any path in $G$ corresponds to an induced path in $L(G)$.

Proof. Suppose $T$ is an induced simple tree in $L(G)$ and $v$ is a vertex of $T$. If $\operatorname{deg}(v) \geq 3$ in $L(G)$, then $v$ and three of its neighbors will induce a $K_{1,3}$ in $L(G)$, contradicting the characterization given in Theorem 3.1. Therefore, every vertex $v$ of $T$ has degree at most two. Thus, $T$ must be an induced path in $L(G)$.

Suppose $e_{1} e_{2} \cdots e_{l-1} e_{l}$ is an induced path in $L(G)$. Since the vertices are distinct in $L(G)$, the edges in $G$ are distinct and the adjacent edges have a common vertex. Thus, $P=v_{1} e_{1} v_{2} e_{2} \cdots v_{l} e_{l} v_{l+1}$ is a path in $G$.

Suppose $P=v_{1} e_{1} v_{2} e_{2} \cdots v_{l} e_{l} v_{l+1}$ is a path in $G$. Then clearly $Q=e_{1} e_{2} \cdots e_{l-1} e_{l}$ is a path in $L(G)$ since adjacent edges in $P$ have a common vertex. In order to show that $Q$ is an induced path in $L(G)$, we show that the vertices $e_{1} e_{2} \cdots e_{l}$ do not induce a cycle amongst themselves. Suppose a cycle is induced, so that $\operatorname{deg}\left(e_{j}\right) \geq 3$, for some $j$ such that $1<j<l$. Then a $K_{1,3}$ is induced in $L(G)$, contradicting that $L(G)$ is a line graph (See Theorem 3.1). If $e_{l}$ and $e_{1}$ are adjacent in $L(G)$ then $v_{l+1}=v_{1}$ in $P$, which contradicts the assertion that the vertices of a path in $G$ are distinct. Therefore, $Q$ is an induced path in $L(G)$.

From Proposition 3.2 we see that finding tree covers of $L(G)$ correspond to finding path decompositions of $G$. A path decomposition of a graph $G$ is a list of paths $\left\{P_{1}, P_{2}, \ldots, P_{k}\right\}$ such that each edge of $G$ appears in exactly one path in the list. The minimum number of paths in such a list is called the path number of $G$ and is denoted by $\pi(G)[12,16]$.

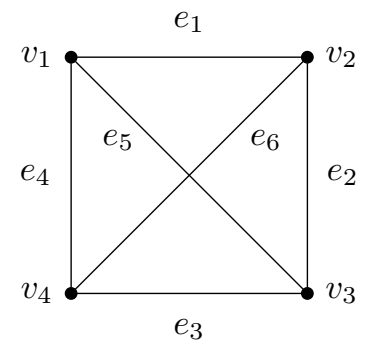

Figure 3 . The complete graph $K_{4}$ can be covered by two paths: $v_{2} v_{1} v_{4} v_{3}$ and $v_{1} v_{3} v_{2} v_{4}$.

THEOREM 3.3. Let $L(G)$ be the line graph of a simple graph $G$. Then $\tau(L(G))=\pi(G)$.

Proof. Let $k$ be the number of paths in a minimal path decomposition of $G$. Each of the paths $P_{1}, \ldots, P_{k}$ in the minimal path decomposition produces induced paths $Q_{1}, \ldots, Q_{k}$ in $L(G)$. Since each edge in $G$ appears in one and only one path in $\left\{P_{1}, \ldots, P_{k}\right\}$, we conclude that each vertex of $L(G)$ appears in one and only one path in $\left\{Q_{1}, \ldots, Q_{k}\right\}$. Thus, we have a vertex disjoint tree cover of $L(G)$ of size $k$. Hence, $\tau(L(G)) \leq k$. By Proposition 3.2, every induced tree in $L(G)$ has to be an induced path in $L(G)$ and every induced path in $L(G)$ is obtained from a path in $G$. Therefore, a minimal tree cover in $L(G)$ must be obtained from a path decomposition in $G$. Hence, $k \leq \tau(L(G))$. Thus, $\tau(L(G))=\pi(G)$.

TheOrem 3.4. Let $L(G)$ be the line graph of a simple graph $G$. Then $\tau(G) \leq \pi(G)=\tau(L(G))$.

Proof. We first show that $\tau(G) \leq \pi(G)$. Let $\left\{T_{1}, \ldots, T_{k}\right\}$ be a minimal tree cover of $G$. If one of the trees $T_{i}$, for some $1 \leq i \leq k$, is not a path, then more than one path is needed to cover the edges of $T_{i}$. Hence, $k \leq \pi(G)$. Now suppose all the trees are induced simple paths in $G$. If all the edges of $G$ are covered by the $k$ paths, then $k=\pi(G)$. If some of the edges of $G$ are not covered by the $k$ paths of the tree cover, then vertices of those edges must induce a cycle with vertices of some path in the cover. Suppose $e$ and $f$ 
Electronic Journal of Linear Algebra, ISSN 1081-3810

A publication of the International Linear Algebra Society

Volume 36, pp. 678-693, September 2020.

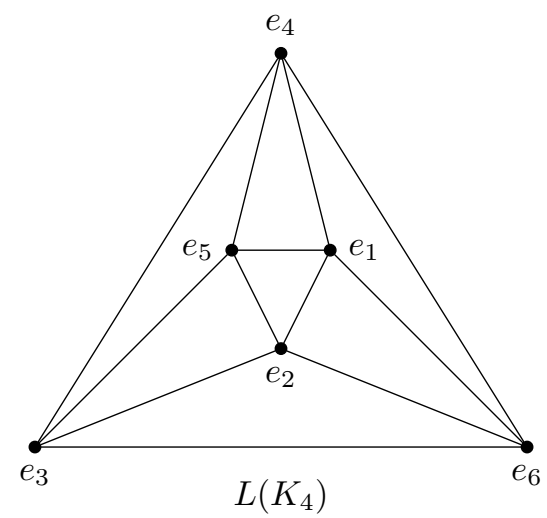

FIGURE 4. Since $\pi\left(K_{4}\right)=2$, where $K_{4}$ is the complete graph, $\tau\left(L\left(K_{4}\right)\right)=2$. The paths $e_{1} e_{4} e_{3}$ and $e_{6} e_{2} e_{5}$ induce a tree cover for $L\left(K_{4}\right)$.

are two edges not covered by the paths $T_{1}, \ldots, T_{k}$. In addition, suppose $e$ and $f$ induce a cycle with some vertices of $T_{1}$. Then at least one additional path is needed to cover edges $e$ and $f$. Hence, $k \leq \pi(G)$. From Theorem 3.3 the conclusion follows. $G$.

Proposition 3.5. If $G$ is a tree, then $\tau(L(G))=\frac{k}{2}$, where $k$ is the number of vertices of odd degree in

Proof. From [16], $\pi(G)=\frac{k}{2}$, where $k$ is the number of vertices of $G$ of odd degree. Thus, $\tau(L(G))=\frac{k}{2}$ using Theorem 3.3.

If $G$ is a tree that is not a path then clearly $\tau(G)=1<\tau(L(G))$. We give below examples of graphs $G$ for which $\tau(G)=\tau(L(G))$.

Proposition 3.6. If a simple graph $G$ is either a path, a cycle, or a complete graph, then $\tau(G)=$ $\tau(L(G))$.

Proof. Since every path $P_{n}$ has two pendant vertices, from Proposition 3.5 we get $\tau\left(P_{n}\right)=1=\tau\left(L\left(P_{n}\right)\right)$.

Suppose the graph $G=C_{n}$, where $C_{n}$ is a cycle on $n$ vertices. Let $V\left(C_{n}\right)=\left\{v_{1}, \ldots, v_{n}\right\}$ with edges $e_{i}=v_{i} v_{i+1}$ for $i=1, \ldots, n-1$ and $e_{n}=v_{1} v_{n}$. Then $\pi\left(C_{n}\right)=2$ with the paths $v_{1} v_{2} \cdots v_{n-1} v_{n}$ and $v_{n} v_{1}$. By Theorem 3.3, $\pi\left(C_{n}\right)=\tau\left(L\left(C_{n}\right)\right)=2$. Also, $\tau\left(C_{n}\right)=2$ with trees consisting of the path $v_{1} v_{2} \cdots v_{n-1}$ and the single vertex $v_{n}$.

Suppose the graph $G=K_{n}$ is a complete graph on $n$ vertices. Let $V\left(K_{n}\right)=\left\{v_{1}, \ldots, v_{n}\right\}$ with edges $v_{i} v_{j}$ for $i \neq j$ and $i, j$ in $\{1,2, \ldots, n\}$. By Theorem 3.4, $\tau\left(K_{n}\right) \leq \pi\left(K_{n}\right)=\tau\left(L\left(K_{n}\right)\right)$. Since every two vertices are adjacent in the graph $K_{n}$, each tree in the tree cover can only contain two vertices without inducing a cycle. Hence, $\tau\left(K_{n}\right)=\left\lceil\frac{n}{2}\right\rceil$. From [12], we have $\pi\left(K_{n}\right)=\left\lceil\frac{n}{2}\right\rceil$.

Proposition 3.7. Let $K_{m, n}$ be a complete bipartite graph. Then

$$
\tau\left(L\left(K_{m, n}\right)\right)= \begin{cases}\frac{m+n}{2} & \text { if } m n \text { is odd } \\ \left\lceil\frac{m n}{2 n-\delta(m, n)}\right\rceil & \text { if } m n \text { even }\end{cases}
$$


Proof. From [12],

$$
\pi\left(K_{m, n}\right)= \begin{cases}\frac{m+n}{2} & \text { if } m n \text { is odd } \\ \left\lceil\frac{m n}{2 n-\delta(m, n)}\right\rceil & \text { if } m n \text { even }\end{cases}
$$

where $\delta(m, n)=1$ if $m=n$ and 0 otherwise. Thus, by Theorem 3.4, we have the result.

3.1. Connection to maximum semidefinite nullity. It has been conjectured in $[2]$ that $\tau(G) \leq$ $M_{+}(G)$. We will verify this conjecture for some classes of line graphs.

It has been shown in [11] that if $G$ is a simple graph with $|G| \geq 2$ and $G$ contains a Hamiltonian path (a path $P$ such that $V(P)=V(G))$, then $m r_{+}(L(G))=|G|-2$. In this case, $M_{+}(L(G))=|E(G)|-|G|+2$. If $G$ is a path $P_{m}$ on $m$ vertices, then $M_{+}\left(L\left(P_{m}\right)\right)=(m-1)-m+2=1=\tau\left(P_{m}\right)=\tau\left(L\left(P_{m}\right)\right)$. If $G$ is a cycle on $m$ vertices, then $M_{+}\left(L\left(C_{m}\right)\right)=m-m+2=2=\tau\left(C_{m}\right)=\tau\left(L\left(C_{n}\right)\right)$.

It has been shown in [11] that if $K_{n}$ is a complete graph on $n$ vertices, then $M_{+}\left(L\left(K_{n}\right)\right)=\frac{n(n-1)}{2}-n+2$. It is not difficult to verify that $\tau\left(L\left(K_{n}\right)\right)=\left\lceil\frac{n}{2}\right\rceil \leq M_{+}\left(L\left(K_{n}\right)\right)$.

It has been shown in [15] that if $T_{n}$ is a tree on $n$ vertices then $m r_{+}\left(L\left(T_{n}\right)\right)=n-l$ where $l$ is the number of pendant vertices in $T_{n}$. Hence, $M_{+}\left(L\left(T_{n}\right)\right)=(n-1)-n+l=l-1$. From Proposition 3.5, we know that $\tau\left(L\left(T_{n}\right)\right)=\frac{k}{2}$ where $k$ is the number of vertices of odd degree. It is a known fact that the degree sum equals twice the number of edges. To see $k \leq 2 l-2$, one can rewrite the left hand side of $\sum_{i=1}^{n}\left(d\left(v_{i}\right)-2\right)=-2$ into three summands: over the set of pendant vertices, the set of even vertices, and the set of odd vertices with degree at least 3 . This results in the inequality $-l+(k-l) \leq-2$.

In the case of the complete bipartite graph $K_{m, n}$, we consider several cases. When $n=1$, then $L\left(K_{m, 1}\right) \equiv$ $K_{m}$. Since $K_{1}$ and $K_{2}$ are both trees, from [17] $M_{+}\left(K_{1}\right)=M_{+}\left(K_{2}\right)=1=\tau\left(L\left(K_{1,1}\right)\right)=\tau\left(L\left(K_{2,1}\right)\right)$. Consider the case $m \geq 3$ and $n=1$. It is well known that $m r_{+}\left(K_{m}\right)=1$. Hence, $M_{+}\left(K_{m}\right)=m-1$. Note that $\frac{m+1}{2} \leq m-1$ and $\left\lceil\frac{m}{2}\right\rceil \leq m-1$ for $m \geq 3$.

From [11], for all complete bipartite graphs $K_{m, n}$ where $m \geq n \geq 2$, we have $m r_{+}\left(L\left(K_{m, n}\right)\right)=m+n-2$ and $M_{+}\left(L\left(K_{m, n}\right)\right)=m n-m-n+2$. We show that $\tau\left(L\left(K_{m, n}\right)\right) \leq M_{+}\left(L\left(K_{m, n}\right)\right)$ holds.

If $m n$ is odd, then $m, n$ must be odd. We may assume $m \geq n>2$. Note that $m>\frac{3}{2}$ and $n>2 \geq \frac{3 m-4}{2 m-3}$. Therefore, we have:

$$
\begin{aligned}
0 & \leq(2 m-3)\left(n-\frac{3 m-4}{2 m-3}\right), \\
0 & \leq 2 m n-3 m-3 n+4, \\
m+n & \leq 2 m n-2 m-2 n+4, \\
\frac{m+n}{2} & \leq m n-m-n+2 .
\end{aligned}
$$

Thus, $\tau\left(L\left(K_{m, n}\right)\right) \leq M_{+}\left(L\left(K_{m, n}\right)\right)$ holds in this case.

Assume $m n$ is even and $m \neq n$. Then we have

$$
\left\lceil\frac{m n}{2 n-\delta(m, n)}\right\rceil=\left\lceil\frac{m n}{2 n}\right\rceil=\left\lceil\frac{m}{2}\right\rceil .
$$


Since

$$
\left\lceil\frac{m}{2}\right\rceil \leq \frac{m+1}{2} \leq \frac{m+n}{2}
$$

by the previous case, the desired inequality holds.

Assume $m n$ even and $m=n$. Then we have

$$
\left\lceil\frac{m n}{2 n-1}\right\rceil=\left\lceil\frac{m^{2}}{2 m-1}\right\rceil=\left\lceil\frac{m^{2}}{m\left(2-\frac{1}{m}\right)}\right\rceil=\left\lceil\frac{m}{2-\frac{1}{m}}\right\rceil,
$$

and consequently,

$$
\left\lceil\frac{m}{2-\frac{1}{m}}\right\rceil \leq\left\lceil\frac{m}{2-\frac{1}{2}}\right\rceil=\left\lceil\frac{2 m}{3}\right\rceil \leq m
$$

Since $m \leq \frac{m+m}{2}$, by the previous case, the desired inequality is satisfied. Therefore, $\tau\left(L\left(K_{m, n}\right)\right) \leq$ $M_{+}\left(L\left(K_{m, n}\right)\right)$.

4. Tree cover number of shadow graphs. Let $G$ be a simple connected graph on two or more vertices. Let $V(G)=\left\{v_{1}, v_{2}, \ldots, v_{n}\right\}$. We add $n$ vertices $\left\{u_{1}, u_{2}, \ldots, u_{n}\right\}$ to $V(G)$ and call $u_{i}$ the shadow vertex of $v_{i}, 1 \leq i \leq n$. We define the shadow graph of $G$, denoted $S(G)$, as follows: the edge set of $S(G)$ is given by $E(G) \cup\left\{u_{i} w: w \in N\left(v_{i}\right), 1 \leq i \leq n\right\}$. In other words, the edge set $S(G)$ consists of all the edges of $G$ along with new edges obtained by joining each shadow vertex $u_{i}(1 \leq i \leq n)$ to the neighbors of $v_{i}$ $(1 \leq i \leq n)$. As an example, if $G=C_{4}$ then $S\left(C_{4}\right)$ is given in Figure 5.

Observe that (1) a vertex of $G$ and its shadow vertex are not adjacent in $S(G)$ and (2) no two shadow vertices are adjacent in $S(G)$. This definition of $S(G)$ appears in [8, p. 276].

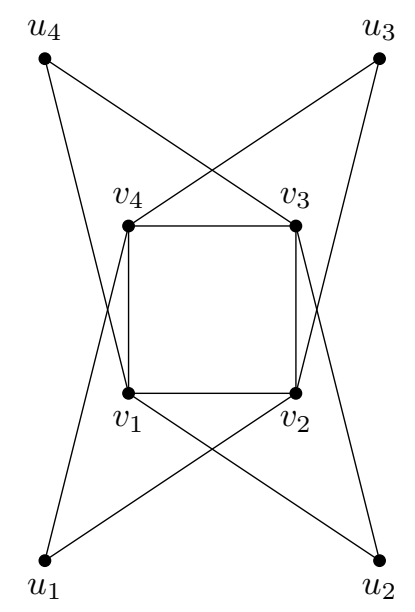

Figure 5. The shadow graph of a cycle $C_{4}$.

We now extend our definition of a shadow graph $S(G)$ to that of a $p$-shadow graph, denoted $S_{p}(G)$. For $p>1$, let $u_{i}^{1}, u_{i}^{2}, \ldots, u_{i}^{p}$ denote the $p$ shadow vertices of $v_{i}, 1 \leq i \leq n$. The edges of $S_{p}(G)$ consists of edges of $G$ along with new edges from each shadow vertex $u_{i}^{1}, u_{i}^{2}, \ldots, u_{i}^{p}$ of $v_{i}$ to the neighbors of $v_{i}$ in $G$, $1 \leq i \leq n$. 
It is clear that if $G$ is a simple connected graph on at least three vertices, then $S(G)$ contains a cycle, and hence, $\tau(S(G)) \geq 2$. In several cases, $\tau(G) \leq \tau(S(G))$. In the case of the path $P_{m}$ on $m$ vertices, we have the strict inequality $\tau\left(P_{m}\right)<\tau\left(S\left(P_{m}\right)\right)$ for $m \geq 3$.

THEOREM 4.1. Let $P_{m}$ be a path on $m$ vertices with $m \geq 3$. Then $\tau\left(S\left(P_{m}\right)\right)=2$. For $p>1$, $\tau\left(S_{p}\left(P_{m}\right)\right)=\left\lceil\frac{m}{2}\right\rceil$.

Proof. Let $V\left(P_{m}\right)=\left\{v_{1}, v_{2}, \ldots, v_{m}\right\}$ and with edges $v_{i} v_{i+1}$ for $i=1, \ldots, m-1$. Let $u_{1}, u_{2}, \ldots, u_{m}$ be the shadow vertices of $v_{1}, v_{2}, \ldots, v_{m}$, respectively.

- Case 1: $m$ is even.

In this case, $v_{1} u_{2} v_{3} u_{4} \cdots v_{m-1} u_{m}$ and $u_{1} v_{2} u_{3} v_{4} \cdots u_{m-1} v_{m}$ are two vertex disjoint induced paths in $S\left(P_{m}\right)$ which cover all the vertices of $S\left(P_{m}\right)$. Hence, $\tau\left(S\left(P_{m}\right)\right) \leq 2$. Since $m \geq 3, \tau\left(S\left(P_{m}\right)\right) \geq 2$.

- Case 2: $m$ is odd.

The paths $v_{1} u_{2} v_{3} u_{4} \cdots u_{m-1} v_{m}$ and $u_{1} v_{2} u_{3} v_{4} \cdots v_{m-1} u_{m}$ are induced in $S\left(P_{m}\right)$ and are vertex disjoint. We conclude $\tau\left(S\left(P_{m}\right)\right)=2$.

Now we consider $S_{p}\left(P_{m}\right)$ for $p>1$.

- Case 1: $m$ is even.

Let $T_{i}$ be the tree induced by the vertices $\left\{v_{i} v_{i+1} u_{i}^{1} \cdots u_{i}^{p} u_{i+1}^{1} \cdots u_{i+1}^{p}\right\}$, where $i=1,3, \ldots, m-1$. These are $\frac{m}{2}$ vertex disjoint induced trees in $S_{p}\left(P_{m}\right)$ that cover all the vertices. Hence, $\tau\left(S_{p}\left(P_{m}\right)\right) \leq$ $\frac{m}{2}$.

- Case 2: $m$ is odd.

For $i=1,3,5, \ldots, m-4$ we use the same trees described in the previous case. There are $\frac{m-3}{2}$ such trees. In addition we consider the two trees induced by

$$
\left\{v_{m-2} v_{m-1} u_{m-2}^{1} \cdots u_{m-2}^{p} u_{m-1}^{1} \cdots u_{m-1}^{p} u_{m}^{1} \cdots u_{m}^{p}\right\}
$$

and by the single vertex $v_{m}$. These $\frac{m-3}{2}+2=\frac{m+1}{2}=\left\lceil\frac{m}{2}\right\rceil$ vertex disjoint induced trees cover all the vertices of $S_{p}\left(P_{m}\right)$. Hence, $\tau\left(S_{p}\left(P_{m}\right)\right) \leq\left\lceil\frac{m}{2}\right\rceil$.

We show that every tree in a minimal tree cover cannot contain more than two adjacent vertices of the path $P_{m}$. Suppose $v_{i-1} v_{i} v_{i+1}$ is a subpath induced in a tree of the tree cover. Then all the shadow vertices $u_{i}^{1}, \ldots, u_{i}^{p}$ have to be isolated vertices in the tree cover as otherwise a cycle would be induced by any one of those shadow vertices along with $v_{i-1} v_{i} v_{i+1}$. Hence, the number of trees in the tree cover is $\left\lceil\frac{m}{3}\right\rceil+p\left\lfloor\frac{m}{3}\right\rfloor \geq(1+p)\left\lfloor\frac{m}{3}\right\rfloor \geq 3\left\lfloor\frac{m}{3}\right\rfloor \geq\left\lceil\frac{m}{2}\right\rceil$. By a similar argument, there will be $\left\lfloor\frac{k}{2}\right\rfloor p\left\lfloor\frac{m}{k}\right\rfloor$ isolated vertices to be covered if we consider induced paths of length $k \geq 3$ in a tree of the tree cover. Therefore, the number of trees in the tree cover is

$$
\left\lceil\frac{m}{k}\right\rceil+\left\lfloor\frac{k}{2}\right\rfloor p\left\lfloor\frac{m}{k}\right\rfloor \geq\left(1+p\left\lfloor\frac{k}{2}\right\rfloor\right)\left\lfloor\frac{m}{k}\right\rfloor \geq\left\lfloor\frac{k}{2}\right\rfloor\left\lfloor\frac{m}{k}\right\rfloor \geq\left\lceil\frac{m}{2}\right\rceil .
$$

Hence, $\left\lceil\frac{m}{2}\right\rceil$ is the minimum number of trees in the tree cover. Therefore, we conclude that $\tau\left(S_{p}\left(P_{m}\right)\right)=$ $\left\lceil\frac{m}{2}\right\rceil$.

In the case of the complete bipartite graph $K_{m, n}$, we show that $\tau\left(K_{m, n}\right)=\tau\left(S\left(K_{m, n}\right)\right)$.

Proposition 4.2. Let $K_{m, n}$ be a complete bipartite graph. Then $\tau\left(S_{p}\left(K_{m, n}\right)\right)=2$.

Proof. Let $V\left(K_{m, n}\right)=\left\{v_{1}, v_{2}, \ldots, v_{m}, w_{1}, w_{2}, \ldots, w_{n}\right\}$ where $\left\{v_{1}, \ldots, v_{m}\right\}$ and $\left\{w_{1}, \ldots, w_{n}\right\}$ are independent sets. The edges of $K_{m, n}$ are $v_{i} w_{j}$ for $1 \leq i \leq m$ and $1 \leq j \leq n$. 
Let $u_{i}^{1}, u_{i}^{2}, \ldots, u_{i}^{p}$ be the $p$-shadow vertices of $v_{i}$ for $1 \leq i \leq m$. Let $y_{j}^{1}, y_{j}^{2}, \ldots, y_{j}^{p}$ be the $p$-shadow vertices of $w_{j}$ for $1 \leq j \leq n$. The graph $S_{p}\left(K_{m, n}\right)$ has vertex set $V\left(K_{m, n}\right) \bigcup\left\{u_{i}^{1}, u_{i}^{2}, \ldots, u_{i}^{p}\right\}_{i=1}^{m} \bigcup\left\{y_{j}^{1}, y_{j}^{2}, \ldots y_{j}^{p}\right\}_{j=1}^{n}$. The edges of $S_{p}\left(K_{m, n}\right)$ are the original edges of $K_{m, n}$ along with edges from each shadow vertex $u_{i}^{1}, \ldots, u_{i}^{p}$ to vertex $w_{a}$ where $i \neq a$ and from each shadow vertex $y_{j}^{1}, \ldots, y_{j}^{p}$ to vertex $v_{b}$ where $j \neq b$.

Then the trees induced by vertices

$$
\left\{v_{1}, y_{1}^{1}, y_{1}^{2}, \ldots, y_{1}^{p}, y_{2}^{1}, y_{2}^{2}, \ldots, y_{2}^{p}, \ldots, y_{m}^{1}, y_{m}^{2}, \ldots, y_{m}^{p}, w_{1}, w_{2}, \ldots, w_{n-1}\right\}
$$

and

$$
\left\{w_{n}, v_{2}, v_{3}, \ldots, v_{n}, u_{1}^{1}, u_{1}^{2}, \ldots u_{1}^{p}, u_{2}^{1}, u_{2}^{2}, \ldots, u_{2}^{p}, \ldots, u_{n}^{1}, u_{n}^{2}, \ldots, u_{n}^{p}\right\}
$$

are vertex disjoint induced trees that cover all vertices of $S_{p}\left(K_{m, n}\right)$.

We show that when $G$ denotes a cycle $C_{n}$ or a complete graph $K_{m}, \tau(G)=\tau(S(G))$ when $|G|$ is even, and $\tau(G)<\tau(S(G))$ when $|G|$ is odd.

THEOREM 4.3. Let $C_{m}$ be a cycle on $m$ vertices with $m \geq 3$. Then

$$
\tau\left(S\left(C_{m}\right)\right)= \begin{cases}2 & \text { if } m \text { is even } \\ 3 & \text { if } m \text { is odd }\end{cases}
$$

Proof. Let $V\left(C_{m}\right)=\left\{v_{1}, v_{2}, \ldots, v_{m}\right\}$, and the cycle has edges $v_{1} v_{m}$ and $v_{i} v_{i+1}$ for $i=1, \ldots, m-1$. Let $u_{1}, \ldots, u_{m}$ be the shadow vertices.

- Case 1: $m$ is even.

In this case, the graphs induced by

$$
\left\{u_{1}, v_{1}, v_{2}, u_{3}, v_{4}, u_{5}, \ldots, u_{m-1}\right\} \text { and }\left\{u_{2}, v_{3}, u_{4}, v_{5}, \ldots, v_{m-1}, v_{m}, u_{m}\right\}
$$

are two vertex disjoint induced trees which cover all the vertices of $S\left(C_{m}\right)$. This shows $\tau\left(S\left(C_{m}\right)\right) \leq 2$. Since $m \geq 3, \tau\left(S\left(C_{m}\right)\right) \geq 2$.

- Case 2: $m$ is odd.

The subgraphs induced by

$$
\left\{u_{1}, v_{2}, u_{3}, v_{4}, \ldots, u_{m-2}, v_{m-1}, u_{m}\right\} \text { and }\left\{u_{2}, v_{3}, u_{4}, v_{5}, \ldots, u_{m-1}, v_{m}\right\}
$$

as well as the isolated vertex $v_{1}$ are three vertex disjoint induced paths which cover all the vertices of $S\left(C_{m}\right)$. Hence, $\tau\left(S\left(C_{m}\right) \leq 3\right.$. Since $m \geq 3, \tau\left(S\left(C_{m}\right)\right) \geq 2$. It remains to show $\tau\left(S\left(C_{m}\right)\right) \neq 2$.

By way of contradiction, assume there exist two vertex disjoint induced trees $T_{1}$ and $T_{2}$ that cover the vertices of $S\left(C_{m}\right)$. Since $\left|S\left(C_{m}\right)\right|=2 m$, we assume without loss of generality $T_{1}$ is induced on at least $m$ vertices. As observed in the proof of Theorem 4.1, suppose $v_{i-1} v_{i} v_{i+1}$ is a path induced in $T_{1}$. Then $u_{i}$ becomes an isolated vertex as otherwise a cycle would be induced by $\left\{u_{i}, u_{i-1}, v_{i}, v_{i+1}\right\}$. This increases the number of trees in the tree cover number to more than two, contradicting our assumption. Since only two adjacent vertices of $C_{m}$ can be included in $T_{1}$, we label them $v_{1}$ and $v_{2}$. The maximum number of vertices in an induced tree $T_{1}$ is $m$ and the tree is induced by $\left\{u_{1}, v_{1}, v_{2}, u_{3}, v_{4}, v_{5}, \ldots, u_{m-2}, v_{m-1}\right\}$. The graph induced in $S\left(C_{m}\right)$ by the remaining vertices has two connected components. Thus, three vertex disjoint, induced trees are required to cover $V\left(S\left(C_{m}\right)\right)$. 
THEOREM 4.4. Let $K_{m}$ be a complete graph on $m$ vertices. Then

$$
\tau\left(S\left(K_{m}\right)\right)= \begin{cases}\left\lceil\frac{m}{2}\right\rceil & \text { if } m \text { is even } \\ \left\lceil\frac{m}{2}\right\rceil+1 & \text { if } m \text { is odd }\end{cases}
$$

Proof. Let $V\left(K_{m}\right)=\left\{v_{1}, v_{2}, \ldots, v_{m}\right\}$ and $E\left(K_{m}\right)=\left\{v_{i} v_{j}: i \neq j\right\}$ for $i, j=1, \ldots, m$. Let $u_{1}, \ldots, u_{m}$ be the shadow vertices of $v_{1}, v_{2}, \ldots, v_{m}$. Note that any three vertices of $K_{m}$ induce a cycle. Thus, any tree in the tree cover of $S\left(K_{m}\right)$ cannot include more than two vertices $v_{i}, v_{j}$ for $i \neq j$ and $i, j \in\{1, \ldots, m\}$. Hence, $\left\lceil\frac{m}{2}\right\rceil \leq \tau\left(S\left(K_{m}\right)\right)$.

Consider any minimal tree cover of $K_{m}$, when $m$ is even. This minimal tree cover consists of $\frac{m}{2}$ edges of $K_{m}$. Thus, $\tau\left(K_{m}\right)=\frac{m}{2}$. Now in $S\left(K_{m}\right)$ consider the $\frac{m}{2}$ trees induced by $\left\{v_{i}, v_{i+1}, u_{i}, u_{i+1}\right\}$, for $i=1,3, \ldots, m-1$. These trees cover all the vertices of $S\left(K_{m}\right)$. Therefore, $\tau\left(S\left(K_{m}\right)\right)=\frac{m}{2}=\left\lceil\frac{m}{2}\right\rceil$.

Consider any minimal tree cover of $K_{m}$, when $m$ is odd. This minimal tree cover consists of $\left\lfloor\frac{m}{2}\right\rfloor$ edges of $K_{m}$ and a single vertex denoted as $v_{1}$. Thus, $\tau\left(K_{m}\right)=\left\lceil\frac{m}{2}\right\rceil$. Now in $S\left(K_{m}\right)$ consider the $\left\lfloor\frac{m}{2}\right\rfloor$ trees induced by $\left\{v_{i}, v_{i+1}, u_{i}, u_{i+1}\right\}$, for $i=2, \ldots, m-1$. These trees cover all the vertices except for $v_{1}$ and $u_{1}$. Now $v_{1}$ and $u_{1}$ have to be isolated vertices in the tree cover of $S\left(K_{m}\right)$ because $v_{i} v_{j} v_{1}$ or $v_{i} v_{j} u_{1}$ for $i \neq j$, $i, j \neq 1$ induce a cycle. Therefore, $\tau\left(S\left(K_{m}\right)\right)=\left\lfloor\frac{m}{2}\right\rfloor+2=\left\lceil\frac{m}{2}\right\rceil+1$.

4.1. Connection to maximum semidefinite nullity. For some classes of shadow graphs, we are able to verify the conjecture that $\tau(G) \leq M_{+}(G)$.

From [17], we know that $m r_{+}(G)=|G|-1$ if and only if $G$ is a tree. Since the shadow graph $S(G)$ of any graph $G$ with at least three vertices contains cycles, we get $m r_{+}(S(G)) \leq|S(G)|-2$. Hence, $M_{+}(S(G)) \geq 2$. Therefore, $\tau\left(S\left(P_{m}\right)\right)=2 \leq M_{+}\left(S\left(P_{m}\right)\right)$ and $\tau\left(S\left(K_{m, n}\right)\right)=2 \leq M_{+}\left(S\left(K_{m, n}\right)\right)$.

Consider $P_{m}$ with $m \geq 3$ and $S_{p}\left(P_{m}\right)$ with $p \geq 1$. Since each shadow vertex has degree at most two, we can sequentially orthogonally remove $u_{i}^{j}$ for a fixed $i$, and $j=1, \ldots, p$. This will not result in any additional edges for $u_{i}^{j}, i=1, m$, since they are all pendent vertices. For the other shadow vertices that all have degree two, new edges have to be drawn between $v_{i-1}$ and $v_{i+1}$ for $i=2, \ldots, m-1$. These new edges produce a graph consisting of $m-2$ triangles. This graph is chordal. The minimum semidefinite rank of the graph is equal to its clique cover number [7]. We count the minimum number of triangles needed to cover all the single edges in the graph.

When there is only one shadow vertex for each vertex of $P_{m}$, the clique cover number is $m-2$ as all edges obtained after orthogonal removal of shadow vertices of degree 2 are single edges. Hence, by [2, 7], $m r_{+}\left(S\left(P_{m}\right)\right)=m+m-2=2 m-2$ and $M_{+}\left(S\left(P_{m}\right)\right)=2 m-(2 m-2)=2$. Thus, $\tau\left(S\left(P_{m}\right)\right)=2=$ $M_{+}\left(S\left(P_{m}\right)\right)$.

Suppose each vertex of $P_{m}$ has $p>1$ shadow vertices. In this case, the orthogonal removal of shadow vertices of degree 2 produces a triangle with a multiedge joining two of its vertices. The $m-2$ triangles can now be covered by $\left\lfloor\frac{m}{2}\right\rfloor$ triangles in its minimum clique cover. Hence, $m r_{+}\left(S_{p}\left(P_{m}\right)\right)=p m+\left\lfloor\frac{m}{2}\right\rfloor$ and $M_{+}\left(S_{p}\left(P_{m}\right)\right)=(p m+m)-\left(p m+\left\lfloor\frac{m}{2}\right\rfloor\right)=\left\lceil\frac{m}{2}\right\rceil=\tau\left(S_{p}\left(P_{m}\right)\right)$.

Suppose $G$ is a cycle $C_{n}$ on $n$ vertices. It can be shown that $3 \leq M_{+}\left(S\left(C_{n}\right)\right)$. To show this, we observe that $K_{4}$ is a minor of $S\left(C_{n}\right)$. Since $\nu\left(K_{s}\right)=s-1$ and $\nu(G)$ is minor-monotone [9, 10] we get $3=\nu\left(K_{4}\right) \leq \nu\left(S\left(C_{n}\right)\right) \leq M_{+}\left(S\left(C_{n}\right)\right)$. Using Theorem 4.3, we now get $\tau\left(S\left(C_{n}\right)\right) \leq M_{+}\left(S\left(C_{n}\right)\right)$. 
Electronic Journal of Linear Algebra, ISSN 1081-3810

A publication of the International Linear Algebra Society

Volume 36, pp. 678-693, September 2020.

5. Tree cover number of corona of two graphs. The corona $G \circ H$ of two simple connected graphs $G$ and $H$ is defined as the graph obtained by taking one copy of $G$ and $|G|$ copies of $H$, and then joining the $i$ th vertex of $G$ to every vertex in the $i$ th copy of $H$.

In the next proposition, we give an upper bound for the tree cover number of $G \circ H$ using the tree cover number of $G$ and the tree cover number of $H$.

Proposition 5.1. If $G$ and $H$ are simple connected graphs then the following inequality holds:

$$
\tau(G \circ H) \leq \tau(G)+|G| \tau(H)
$$

Proof. Let $T_{1}, T_{2}, \ldots, T_{k}$ be a minimum tree cover of $G$ and $M_{1}, M_{2}, \ldots, M_{l}$ be a minimum tree cover of $H$. Taking $|G|$ copies of $M_{i}, 1 \leq i \leq l$, together with $T_{1}, T_{2}, \ldots, T_{k}$ form a tree cover of $G \circ H$. Hence, $\tau(G \circ H) \leq \tau(G)+|G| \tau(H)$.

In Theorem 5.2 we show that equality is possible in Proposition 5.1 when $H$ is a tree.

TheOREM 5.2. If $G$ is a simple connected graph and $H$ is a tree, then $\tau(G \circ H)=\tau(G)+|G|$.

Proof. Let $G$ be a graph on $n$ vertices, denoted $\left\{v_{1}, v_{2}, \ldots, v_{n}\right\}$. Let $H$ be a tree on $m$ vertices, denoted $\left\{w_{1}, w_{2}, \ldots, w_{m}\right\}$ for $m \geq 2$. Consider the corona graph $C=G \circ H$. Then

$$
V(C)=\left\{v_{1}, v_{2}, \ldots, v_{n}, w_{1}^{1}, w_{2}^{1}, \ldots, w_{m}^{1}, \ldots, w_{1}^{n}, w_{2}^{n}, \ldots, w_{m}^{n}\right\}
$$

where $w_{i}^{j}, i \in\{1,2, \ldots, m\}$, corresponds to the vertex $v_{j}$ in the $j$-th copy of $H$. In $C$, each vertex $v_{j}$ of $G$ for $j \in\{1, \ldots, n\}$ is connected to a copy of $H$ by edges $v_{j} w_{i}^{j}$ for $i=\{1,2, \ldots, m\}$. It is clear $\tau(H)=1$. By Proposition 5.1, $\tau(C) \leq \tau(G)+|G| \tau(H)=\tau(G)+|G|$.

We need to show that a smaller tree cover for $C$ cannot be obtained. The additional edges between each vertex $v_{j}$ of $G$ and the vertices in a copy of $H$ do not reduce the number of trees needed to cover $G$ as an induced subgraph. This is because these edges do not connect vertices of $G$ to one another, nor copies of $H$ to other copies of $H$. Thus, any smaller tree cover would need to connect the copies of $H$ to its corresponding vertex in the tree cover of $G$.

Consider a tree in the tree cover of $C$, say $T^{j}$ that covers the vertex $v_{j}$. We want to extend this tree to vertices in the $j$-th copy of $H$. There are a few options for adding vertices of the tree $H$ to the tree $T^{j}$. Any pendant vertex of the tree $H$ can be added to $T^{j}$ without inducing a cycle. Note, if $w_{i}^{j}$ is a pendant, $w_{i}^{j}, v_{j}$, and any neighbor of $w_{i}^{j}$ will induce a cycle, so no neighbor of the pendant vertex can be added to $T^{j}$. Adding pendant vertices to $T^{j}$ does not decrease the tree cover number since not every vertex of $H$ can be added.

Instead, consider adding a non-pendant vertex, $w_{i}^{j}$ to $T^{j}$. By the same argument as above, no neighbor of $w_{i}^{j}$ can then be added to $T^{j}$ or a cycle will be induced. Thus, adding $w_{i}^{j}$ to $T^{j}$ increases the tree cover number since the remaining vertices of this copy of $H$ now requires more than one tree to cover the vertices. Thus, $\tau(C)=\tau(G)+|G|$.

The example in Figure 6 shows that for $G=K_{2}$ and $H=C_{4}, \tau(G \circ H)=3$ whereas $\tau(G)+|G| \tau(H)=5$. Thus, strict inequality is possible in Proposition 5.1.

5.1. Connection to maximum semidefinite nullity. We verify the conjecture that $\tau(G \circ H) \leq$ $M_{+}(G \circ H)$ in the case that both $G$ and $H$ independently satisfy the conjecture. 

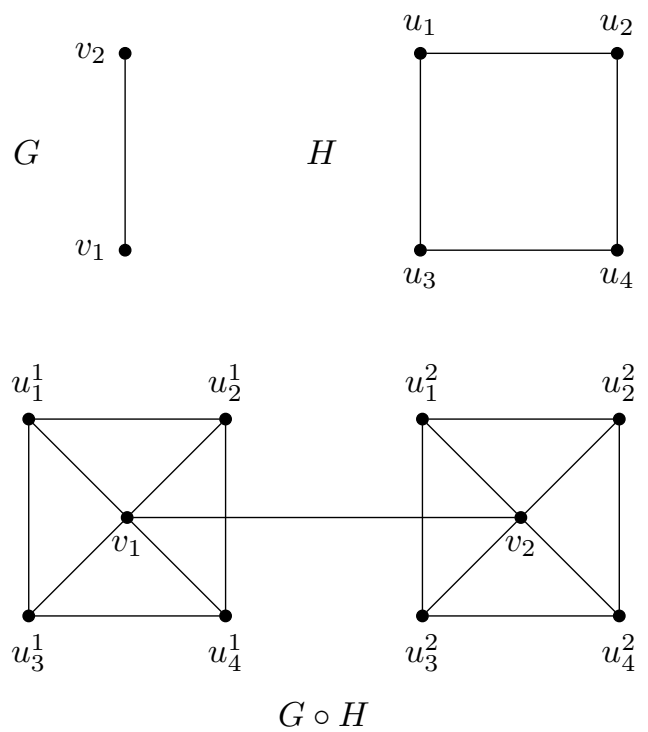

Figure 6. Here, $V(G)=\left\{v_{1}, v_{2}\right\}$ and $V(H)=\left\{u_{1}, u_{2}, u_{3}, u_{4}\right\}$. The corona (given below) has $\tau(G \circ H)=3<5=$ $\tau(G)+|G| \tau(H)$. The three induced trees are $u_{4}^{1} v_{1} v_{2} u_{3}^{2}, u_{3}^{1} u_{1}^{1} u_{2}^{1}$, and $u_{1}^{2} u_{2}^{2} u_{4}^{2}$.

If $G$ is a graph with a cut vertex $v$, such that $H_{1}$ and $H_{2}$ are the connected components of the vertex deletion $G-v$, then we write $G=G_{1} \cdot G_{2}$ where $G_{1}$ and $G_{2}$ are the subgraphs of $G$ induced by $V\left(H_{1}\right) \cup\{v\}$ and $V\left(H_{2}\right) \cup\{v\}$, respectively.

It was shown in [6] that if $G=G_{1} \cdot G_{2}$, then $m r_{+}(G)=m r_{+}\left(G_{1}\right)+m r_{+}\left(G_{2}\right)$. From this, we see that $m r_{+}(G \circ H)=m r_{+}(G)+|G| m r_{+}(H)$. Thus, $M_{+}(G \circ H)=M_{+}(G)+|G| M_{+}(H)$.

From Proposition 5.1, we know that $\tau(G \circ H) \leq \tau(G)+|G| \tau(H)$. Therefore, if $G$ and $H$ satisfy $\tau(G) \leq M_{+}(G)$ and $\tau(H) \leq M_{+}(H)$, then $\tau(G \circ H) \leq \tau(G)+|G| \tau(H) \leq M_{+}(G)+|G| M_{+}(H)=M_{+}(G \circ H)$.

6. Tree cover number of Cartesian products of two graphs. The Cartesian product of simple graphs $G$ and $H$ is the graph $G \square H$ whose vertex set is $V(G) \times V(H)$ and whose edge set is the set of all pairs $\left\{\left(u_{1}, v_{1}\right),\left(u_{2}, v_{2}\right)\right\}$ such that $\left(u_{1} u_{2} \in E(G)\right.$ and $\left.v_{1}=v_{2}\right)$ or $\left(u_{1}=u_{2}\right.$ and $\left.v_{1} v_{2} \in E(H)\right)$. The vertices of a Cartesian product can be viewed in a grid, where the $i$-th row consists of all vertices with form $\left(\cdot, v_{i}\right)$ and induces a copy of the graph $G$, and the $j$-th column consists of all vertices with form $\left(u_{j}, \cdot\right)$ and induces a copy of the graph $H$. For example, the Cartesian product $P_{m} \square P_{n}$ of two paths $P_{m}$ and $P_{n}$ is the $(m \times n)$-grid. We obtain the following upper bound for the tree cover number of $G \square H$.

Proposition 6.1. Let $G$ and $H$ be simple connected graphs. Then

$$
\tau(G \square H) \leq \min \{|G| \tau(H),|H| \tau(G)\} .
$$

Proof. Let $G$ and $H$ be simple connected graphs. Consider the graph $G \square H$. Draw the vertices of graph $G \square H$ as a grid where each row of vertices induce a copy of the graph $G$ and each column of vertices induce a copy of the graph $H$. Since no additional edges are added between two vertices in the same row, $G \square H$ can be covered by the following tree cover: in each row, use the same tree cover as the minimum tree cover of $G$. This results in $|H| \tau(G)$ trees. Similarly, since no additional edges are added between two vertices in the same column, $G \square H$ can be covered by the following tree cover: in each column, use the same tree cover 
Electronic Journal of Linear Algebra, ISSN 1081-3810

A publication of the International Linear Algebra Society

Volume 36, pp. 678-693, September 2020.
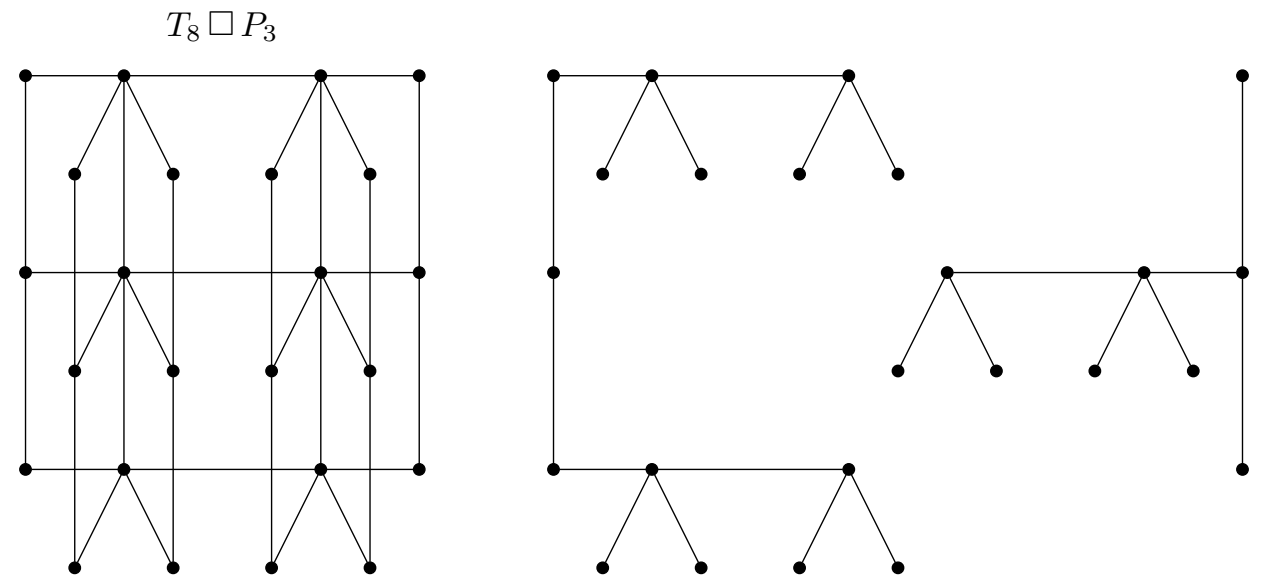

Figure 7. The above graph illustrates Theorem 6.2. The graph $T_{8} \square P_{3}$ can be covered by two vertex disjoint induced trees, displayed as induced subgraphs to the right of $T_{8} \square P_{3}$.

as the minimum tree cover of $H$. This results in $|G| \tau(H)$ trees. The minimum of these two quantities gives an upper bound for $\tau(G \square H)$.

The following results show that strict inequality is possible in Proposition 6.1.

THEOREM 6.2. Let $T_{m}$ and $T_{n}$ be trees on $m \geq 2$ and $n \geq 2$ vertices respectively. Then $\tau\left(T_{m} \square T_{n}\right)=2$.

Proof. Let $V\left(T_{m}\right)=\left\{u_{1}, u_{2}, \ldots, u_{m}\right\}$ and $V\left(T_{n}\right)=\left\{v_{1}, v_{2}, \ldots, v_{n}\right\}$. The vertices of $T_{m} \square T_{n}$ are $\left\{\left(u_{i}, v_{j}\right): 1 \leq i \leq m, 1 \leq j \leq n\right\}$. Since every tree must have at least two pendant vertices, we consider a longest path in $T_{m}$ with endpoints $a$ and $b$. We label $T_{m}$ such that $u_{1}=a$ and $u_{m}=b$. Since any tree is bipartite, we can partition the vertices of $T_{n}$ into two partite sets, $H_{1}$ and $H_{2}$.

Let $G_{1}$ be the tree induced by the vertices in the right-most column of the $m \times n$ grid along with the vertices in the $i$-th rows, where $v_{i} \in H_{1}$, that are not in the left-most column of the grid. Let $G_{2}$ be the tree induced by the remaining vertices of $T_{m} \square T_{n}$.

No cycles are induced between vertices in distinct rows of $G_{1}$ (or $G_{2}$ ) because of the vertices $\left(u_{i}, v_{j}\right.$ ), $\left(u_{i}, v_{k}\right)$ in $G_{1}$ have $v_{j}$ and $v_{k}$ in the same partite set. No cycles are induced between vertices in distinct columns of $G_{1}$ (or $G_{2}$ ) because each row represents the tree $T_{m}$ which is acyclic. Therefore, $\tau\left(T_{m} \square T_{n}\right) \leq 2$. Since $\tau\left(T_{m} \square T_{n}\right)>1$, the theorem holds.

Proposition 6.3. Let $C_{m}$ be a cycle on $m$ vertices and $P_{n}$ be a path on $n$ vertices. Then

$$
\tau\left(C_{m} \square P_{n}\right)=2 .
$$

Proof. The two trees described in Theorem 6.2 cover the vertices of $C_{m} \square P_{n}$. Since the vertices of the first column are in one tree and the vertices of the last column are in another tree, a cycle $C_{m}$ cannot be induced. Thus, $\tau\left(C_{m} \square P_{n}\right)=2$.

THEOREM 6.4. Let $P_{m}$ be a path on $m$ vertices and $K_{n}$ be a complete graph on $n$ vertices. Then $\tau\left(K_{n} \square P_{m}\right)=\left\lceil\frac{n}{2}\right\rceil$.

Proof. The $n m$ vertices $\left(v_{i}, w_{j}\right)$ of $K_{n} \square P_{m}$ consists of the vertices $\left\{v_{1}, \ldots, v_{n}\right\}$ of $K_{n}$ and the vertices $\left\{w_{1}, \ldots, w_{m}\right\}$ of $P_{m}$. Each row of $K_{n} \square P_{m}$ corresponds to $K_{n}$ and each column corresponds to $P_{m}$. Each 
induced tree in $K_{n} \square P_{m}$ contains at most two vertices from each row as otherwise a cycle will be induced. Thus, $\tau\left(K_{n} \square P_{m}\right) \geq\left\lceil\frac{n}{2}\right\rceil$. To prove equality, it suffices to describe a tree cover of size $\left\lceil\frac{n}{2}\right\rceil$.

- Case 1: $n$ is even.

Let $T_{i}$ be the induced graph on vertices $\{(i, 1),(i+1,1),(i+1,2),(i+2,2), \ldots,(i+m-1(\bmod n), m)$, $(i+m(\bmod n), m)\}$ for $i=1,3,5, \ldots, n-1$. Then $T_{1}, T_{3}, \ldots, T_{n-1}$ are $\frac{n}{2}$ vertex disjoint simple trees which cover $K_{n} \square P_{m}$.

- Case 2: $n$ is odd.

In the first $n-1$ columns, cover the vertices as above in the even case. Cover the vertices of the $n$-th column using a path. Hence, $\tau\left(K_{n} \square P_{m}\right) \leq \frac{n-1}{2}+1=\left\lceil\frac{n}{2}\right\rceil$.

6.1. Connection to maximum semidefinite nullity. From [17], we know that $m r_{+}(G)=|G|-1$ if and only if $G$ is a tree. Therefore, $M_{+}(G)=1$ if and only if $G$ is a tree. Since $P_{m} \square P_{n}, C_{m} \square P_{n}$ and $T_{n} \square P_{m}$ are not tree graphs, their maximum semidefinite nullity is at least two. Therefore, $\tau\left(P_{m} \square P_{n}\right) \leq$ $M_{+}\left(P_{m} \square P_{n}\right), \tau\left(C_{m} \square P_{n}\right) \leq M_{+}\left(C_{m} \square P_{n}\right)$ and $\tau\left(T_{n} \square P_{m}\right) \leq M_{+}\left(T_{n} \square P_{m}\right)$. From [15], we know that for $n \geq 2, M_{+}\left(K_{n} \square P_{m}\right)=n$. Thus, $\tau\left(K_{n} \square P_{m}\right)=\left\lceil\frac{n}{2}\right\rceil<n=M_{+}\left(K_{n} \square P_{m}\right)$.

7. Conclusions. The tree cover number for some graph classes involving line graphs, shadow graphs, corona of two graphs, and Cartesian products has been found and verified. It has also been shown that these classes satisfy the conjecture described in [2] that $\tau(G) \leq M_{+}(G)$. These results along with the results in [2] provide additional strong evidence in support of this conjecture.

Acknowledgment. The first author would like to thank Central Michigan University for providing support. The authors thank the referee for the suggestion to add an additional result.

\section{REFERENCES}

[1] F. Barioli, W. Barrett, S.M. Fallat, H.T. Hall, L. Hogben, B. Shader, P. van den Driessche, and H. van der Holst. Zero forcing parameters and minimum rank problems. Linear Algebra and its Applications, 433:401-411, 2010.

[2] F. Barioli, S.M. Fallat, L.H. Mitchell, and S.K. Narayan. Minimum semidefinite rank of outerplanar graphs and the tree cover number. Electronic Journal of Linear Algebra, 22:10-21, 2011.

[3] L.W. Beineke. Derived graphs and digraphs. Beiträge zur Graphentheorie, 17-33, 1968.

[4] L.W. Beineke. Characterizations of derived graphs. Journal of Combinatorial Theory, 9:129-135, 1970.

[5] J.A. Bondy and U.S.R. Murty. Graph Theory (Graduate Texts in Mathematics). Springer, New York, 2008.

[6] M. Booth, P. Hackney, B. Harris, C.R. Johnson, M. Lay, T.D. Lenker, L.H. Mitchell, S.K. Narayan, A. Pascoe, and B.D. Sutton. On the minimum semidefinite rank of a simple graph. Linear and Multilinear Algebra, 59:483-506, 2011.

[7] M. Booth, P. Hackney, B. Harris, C.R. Johnson, M. Lay, L.H. Mitchell, S.K. Narayan, A. Pascoe, K. Steinmetz, B.D. Sutton, and W. Wang. On the minimum rank among positive semidefinite matrices with a given graph. SIAM Journal on Matrix Analysis and Applications, 30:731-740, 2008.

[8] G. Chartrand and P. Zhang. A First Course in Graph Theory. Dover Publications, New York, 2012.

[9] Y.C. de Verdière. On a new graph invariant and a criterion for planarity. Contemporary Mathematics, 147:137-147, 1993.

[10] Y.C. de Verdière. Multiplicities of eigenvalues and tree-width of graphs. Journal of Combinatorial Theory, Series B, $74: 121-146,1998$.

[11] S.M. Fallat and A. Soltani. Line graphs: Their maximum nullities and zero forcing numbers. Czechoslovak Mathematical Journal, 66:743-755, 2016.

[12] F. Harary and A. J. Schwenk. Evolution of the path number of a graph, covering and packing in graphs II. In: R.C. Read (editor), Graph Theory and Computing, 39-45, 1972.

[13] R.A. Horn and C.R. Johnson. Matrix Analysis, second edition. Cambridge University Press, Cambridge, 2013. 
Electronic Journal of Linear Algebra, ISSN 1081-3810

A publication of the International Linear Algebra Society

$\left[\begin{array}{l}\mathrm{IL} \\ \mathrm{AS}\end{array}\right]$

693

Tree Cover Number and Maximum Semidefinite Nullity of Some Graph Classes

[14] J. Krausz. Demonstration nouvelle d'une thorme de whitney sur les rseaux. Math. Fiz., 50:75-85, 1943.

[15] T.A. Peters. Positive semidefinite maximum nullity and zero forcing number. Electronic Journal of Linear Algebra, 23:815-830, 2012.

[16] R.G. Stanton, D.D. Cowan, and L.O. James. Some results on path numbers. Proceedings of Louisiana Conference on Combinatorics, Graph Theory and Computing, 112-135, 1970.

[17] H. van der Holst. Graphs whose positive semi-definite matrices have nullity at most two. Linear Algebra and its Applications, 375:1-11, 2003.

[18] A.C.M. van Rooij and H.S. Wilf. The interchange graph of a finite graph. Acta Mathematica Hungarica, 16:263-269, 1965.

[19] D.B. West. Introduction to Graph Theory, second edition. Prentice Hall, Inc., Upper Saddle River, NJ, 2001. 